\title{
TSP-1 as a novel biological marker of tumor vasculature normalization in colon carcinoma induced by Endostar
}

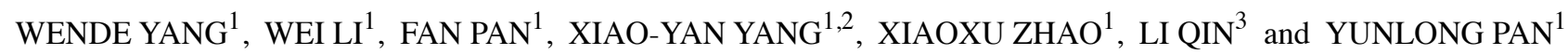 \\ ${ }^{1}$ Department of General Surgery, The First Affiliated Hospital of Jinan University; ${ }^{2}$ Key Laboratory of \\ Functional Protein Research of Guangdong Higher Education Institutes, Institute of Life and Health Engineering, \\ College of Life Science and Technology, Jinan University; ${ }^{3}$ Departments of Histology and Embryology, \\ Medical School of Jinan University, Guangzhou, Guangdong 510632, P.R. China
}

Received January 30, 2018; Accepted May 16, 2019

DOI: $10.3892 / \mathrm{ol} .2020 .11320$

\begin{abstract}
Blood vessels in tumors often exhibit abnormal morphology and function, which promotes the growth, metastasis and resistance of tumors to conventional therapies. Therefore, vascular normalization is an emerging strategy to enhance the effectiveness of radiotherapy and chemotherapy when used in combination; however, there is a lack of evidence regarding the optimal schedule for the co-administration of anti-angiogenic and chemotherapeutic drugs. Scheduling treatment is important as the period for normalization is transient, also known as the 'time window'; however, no biomarker has been identified to detect this window. In the present study, recombinant human endostatin (rhES) was employed as an anti-angiogenic agent in xenograft tumor tissue in mice. Following rhES or control (saline) treatment, the density and integrity of tumor vessels were detected by immunofluorescence staining for cluster of differentiation 31 and $\alpha$-smooth muscle actin; the level of hypoxia in tumor tissue was examined by immunohistochemistry with pimonidazole; the necrotic area was evaluated by hematoxylin and eosin staining; and the level of thrombospondin-1 (TSP-1) in plasma was tested by ELISA. The Cell Counting Kit- 8 assay was also used to evaluate the effect of rhES on the proliferation of colon carcinoma SW620 cells. A 'time window' normalized vasculature was determined between day 4 and 6 following rhES treatment, and accompanied by a decrease in hypoxia in tumor tissue. Decreasing plasma TSP-1 levels were consistent with changes in vascular morphology and hypoxia, which exhibited features of normalization. In addition, rhES had no effect on the proliferation of SW620 cells, suggesting that the reduction in TSP-1 was associated with increased oxygen content during
\end{abstract}

Correspondence to: Professor Yunlong Pan, Department of General Surgery, The First Affiliated Hospital of Jinan University, 613 Huangpu Avenue, Guangzhou, Guangdong 510632, P.R. China E-mail: tpanyl@jnu.edu.cn

Key words: tumor vascular normalization, angiogenesis, thrombospondin-1, recombinant human endostatin, colon carcinoma vascular normalization, rather than inhibited cell proliferation. In conclusion, TSP-1 may be a potential biomarker for predicting the normalization window of colon cancer vessels.

\section{Introduction}

Angiogenesis is a key factor associated with the development of solid tumors, being required to provide adequate nutrition and prompt waste disposal in the tumor microenvironment. Angiogenesis may also serve a role in the shedding of neoplastic cells in the initial stage of metastasis (1). The idea that anti-angiogenic therapies may be used to repress the supply of nutrients and oxygen in order to starve tumor cells and reduce metastasis was first reported by Folkman (1), who proposed the novel theory of inhibiting tumorigenesis via an anti-angiogenic approach. However, attempts to de-vascularize tumors may result in the exacerbation of hypoxia and acidosis within the tumor microenvironment $(1,2)$, an event which reportedly enhances chemoresistance $(3,4)$, reduces the efficacy of radiotherapy $(5,6)$, increases tumor cell malignancy and promotes metastasis $(2,7,8)$. Considering these issues, Jain (9) proposed that the tumor vasculature undergoes a transition from an abnormal to normal state following anti-angiogenic therapy, which may improve blood vessel structure and function. Therefore, treatment with anti-angiogenic therapy may reduce the hypoxic regions within tumors and increase tumor perfusion $(9,11)$. This may allow enhanced delivery of cytotoxic agents to the tumor via improved blood vessel structure and function, improving the efficacy of radiotherapy $(3-7,10,11)$.

However, the period in which the tumor vasculature becomes normalized is transient; this 'time window' may be associated with the balance of anti-angiogenic and pro-angiogenic factor activation, which affects drug resistance $(9,11)$. Thus, identifying the duration of tumor vasculature normalization is crucial. In addition, patients can demonstrate varying sensitivities to anti-angiogenic therapies, and some patients may experience severe side effects, including hemorrhage and thromboembolism (12). Therefore, identifying biomarkers associated with vasculature normalization may prove useful in the determination of which patients may benefit from anti-angiogenic therapy, as well as in the optimization of treatment with anti-tumor therapies (12). 
Several studies have investigated the application of the biomarkers associated with anti-angiogenic therapy: Schneider et al (13) suggested that vascular endothelial growth factor (VEGF)/VEGF receptor 2 (VEGFR2) genotypes may be used to predict the therapeutic efficacy and toxicity of bevacizumab in patients with advanced breast cancer. In addition, findings have suggested that soluble VEGFR1 (also known as sFlt1), which inhibits VEGF activity, may be a promising biomarker of vascular normalization (14). However, these effects do not appear to be associated with solid tumor vasculature normalization, which is characterized by decreased regions of hypoxia and decreased interstitial fluid pressure (IFP) $(9,14)$. The IFP of tumors can be monitored; however, a tissue puncture examination cannot be performed, as this may promote the metastasis of tumor cells. Recently, Lassau et al (15) used dynamic contrast enhanced ultrasonography to observe tumor vascular normalization. This method appeared to be suitable for clinical use for measuring the degree of tumor blood perfusion over time.

Thrombospondin-1 (TSP-1) was the first characterized endogenous angiogenesis inhibitor, which induces the apoptosis and inhibits the migration of endothelial cells by binding to cluster of differentiation (CD)36 and CD148 (16-18). Metronomic chemotherapy can upregulate TSP-1 expression and maintain the balance between pro- and anti-angiogenic factors; and thus tumor vasculature normalization may be induced (19). Firlej et al (20) reported that high expression of TSP-1 in prostate cancer may inhibit angiogenesis; however, tumor progression may be promoted. A major concern is that anti-angiogenic therapies could excessively aggravate hypoxia and stimulate the migration of cancer cells (2). It has been reported that hypoxia modifies calcium homeostasis in prostate carcinoma C4-2 cells and may also induce the expression of TSP-1 (20). Tumor vasculature normalization may create a transient condition that alleviates hypoxia (9). Thus, TSP-1 expression levels in the blood may be used to monitor the vascular normalization 'time window'. In the present study, recombinant human endostatin (rhES) was employed as an anti-angiogenic agent to evaluate the association between TSP-1 expression levels and tumor vascular normalization.

\section{Materials and methods}

Cell isolation and culture. The colon carcinoma cell line SW620 was purchased from the Cell Bank of Type Culture Collection of Chinese Academy of Sciences (Shanghai, China) and was cultured in Dulbecco's modified Eagle's medium at $37^{\circ} \mathrm{C}$ in $5 \% \mathrm{CO}_{2}$. (Gibco; Thermo Fisher Scientific, Inc., Waltham, MA, USA) supplemented with $10 \%$ fetal bovine serum (Gibco; Thermo Fisher Scientific, Inc.) and $100 \mathrm{U} / \mathrm{ml}$ penicillin-streptomycin.

Animal care and tumor establishment. The present study was approved by the Laboratory Animal Ethics Committee of Jinan University (Guangzhou, China). Female BALB/c $(\mathrm{nu} / \mathrm{nu})$ mice $(\mathrm{n}=40 ; 4$ weeks-old; mean body weight, $18.0 \pm 2.0 \mathrm{~g}$ ) were obtained from Beijing HFK Bioscience Co., Ltd. (Beijing, China), and were maintained under specific pathogen-free conditions with food and water provided ad libitum. The institutional laboratory housing temperature was maintained between 23 and $25^{\circ} \mathrm{C}$ and the relative humidity was maintained between 45 and $60 \%$ with a 12 -h light/dark cycle. Prior to implantation of tumorigenic cells, blood was obtained from the corner of the eye of all mice, with 50-100 $\mu \mathrm{l}$ obtained each time to determine the basal expression levels of TSP-1. Xenograft tumors were generated via subcutaneous injection of SW620 cells ( $3 \times 10^{6}$ cells/mouse) into the right flank. Tumor size was measured daily using Vernier calipers, and the volume $(\mathrm{V})$ was calculated as follows: $\mathrm{V}=\left(\right.$ length $\mathrm{x}$ width $\left.{ }^{2}\right)$ $\mathrm{x} 0.523$. Treatment with rhES $[5 \mathrm{mg} / \mathrm{kg}$, diluted with normal saline (NS) to a final volume of $150 \mu \mathrm{l} ; \mathrm{n}=20]$ was applied via an intraperitoneal injection when the tumor size ranged between $100-150 \mathrm{~mm}^{3}$. The same volume of NS was injected as a control $(n=20)$. Reagents were administered continuously for 4 days, and then every 2 days by single injections. Tumor tissues were collected on days $0,2,4,6$ and 12 and the extent of tumor vascular normalization was determined.

Hypoxia inducible factor (HIF)-1 / /TSP-1 detection. Tumor samples $(100 \mu \mathrm{g})$ were ground using a tissue grinder, then centrifuged at $14,000 \mathrm{x}$ g for $30 \mathrm{~min}$ at $4^{\circ} \mathrm{C}$ and the expression levels of HIF-1 $\alpha$ were detected by western blotting. The levels of mouse TSP-1 in the serum were quantified using an ELISA kit (Abcam, Cambridge, UK) according to the manufacturer's protocol, plasma TSP-1 detection was performed 7 days before tumor implantation, on the day of tumor implantation, and $2,4,6,9$ and 12 days after tumor implantation.

Immunohistochemical analysis. Tissues were fixed in $4 \%$ paraformaldehyde at room temperature for $24 \mathrm{~h}$ and then paraffin-embedded, slices were sectioned $6 \mu \mathrm{m}$ thick, deparaffinized in xylene for $10 \mathrm{~min}$, and rehydrated via a graded alcohol gradient $(100,85$ and $75 \%)$ and $\mathrm{ddH}_{2} \mathrm{O}$, each for $5 \mathrm{~min}$. Samples were then subjected to antigen retrieval at $95^{\circ} \mathrm{C}$ using citric acid buffer ( $\mathrm{pH}$ 6.0). Sections were incubated with a solution of $3 \% \mathrm{H}_{2} \mathrm{O}_{2}$ in methanol for $30 \mathrm{~min}$, and rinsed twice with tris-buffered saline (TBS; 5 min per rinse). Subsequently, sections were blocked in 5\% normal goat serum (Beyotime Institute of Biotechnology, Haimen, China) in TBS for $1 \mathrm{~h}$ at room temperature. The sections were then incubated with mouse monoclonal anti-pimonidazole antibody (1:50; HP1-200; Hypoxyprobe, Burlington, MA, USA) in TBS at $4^{\circ} \mathrm{C}$ overnight. Sections were washed by TBS as aforementioned, then incubated with biotin-conjugated anti-mouse secondary antibody (1:200; A0286; Beyotime Institute of Biotechnology, Haimen, China) in TBS for $1 \mathrm{~h}$ at room temperature. Following rinsing, streptavidin-peroxidase was applied for $15 \mathrm{~min}$ at room temperature; the sections were developed with the chromogen 3,3'-diaminobenzidine at room temperature. The sections were dehydrated with graded alcohol (75, 85 and 100\%) for $5 \mathrm{~min}$, transferred to xylene for $5 \mathrm{~min}$ and then air dried for $30 \mathrm{~min}$ prior to mounting. Images were obtained using a Leica DM6000B microscope (Leica Microsystems GmbH, Wetzlar, Germany).

Immunofluorescence analysis. Tissues were fixed, paraffinembedded, sectioned, dewaxed, rehydrated and subjected to antigen retrieval as aforementioned. The sections were blocked in $2 \%$ normal goat serum for $1 \mathrm{~h}$ and stained with the following primary antibodies: Anti-CD31 (1:500; ab28364, Abcam) and anti- $\alpha$-smooth muscle actin ( $\alpha$-SMA; $1: 100$; 
14395-1-AP, Proteintech Group Inc., Rosemont, IL, USA), for labeling of the endothelium and pericytes, respectively. The sections were then washed with TBS and incubated with rhodamine-conjugated goat anti-rat immunoglobulin $(\mathrm{Ig}) \mathrm{G}(\mathrm{H}+\mathrm{L})$ antibody (1:50; SA00007-7, Proteintech) or goat anti-rabbit IgG-fluorescein isothiocyanate antibody (1:200; sc-2012, Santa Cruz Biotechnology, Inc., Dallas, TX, USA) for $40 \mathrm{~min}$ at room temperature. The degree of pericyte detection was presented as a percentage of the length along $\mathrm{CD} 31^{+}$vessels.

Hematoxylin and eosin staining. The initial processing steps were the performed as aforementioned. Slides were flamed and immersed in xylene for $15 \mathrm{~min}$, which was then repeated prior to hydrating the tissue sections via a decreasing series of graded alcohol $(100,90,80$ and $70 \%)$ and water. The sections were stained in hematoxylin for $5 \mathrm{~min}$ at room temperature, and then washed in running tap water for $5 \mathrm{~min}$. The slides were placed in $1 \%$ acid alcohol $(1 \% \mathrm{HCl}$ in $70 \%$ alcohol) for $3 \mathrm{~min}$, and washed in running tap water. The slides were then stained in $1 \%$ eosin $\mathrm{Y}$ for $5 \mathrm{~min}$ at room temperature and washed in tap water for 1-5 min, then dehydrated in increasing concentrations of alcohol $(75,85$ and $100 \%)$ and cleared in xylene. Sections were cut into $6 \mu \mathrm{m}$, and then examined under a light microscope (Leica DM6000B) at x20 magnification. Necrotic regions were determined using ImageJ software (version 1.49; National Institutes of Health, Bethesda, MA, USA).

Western blotting. For western blot analysis, cells or flesh tissues were lysed on ice using PIPA buffer (P0013B; Beyotime Institute of Biotechnology, Haimen, Chain) supplemented with PMSF (ST506, Beyotime, Haimen, China). Concentrations were determined using a BCA protein assay kit (P0010S; Beyotime Institute of Biotechnology,Haimen, China). Proteins were loaded at a mass of $30 \mu \mathrm{g}$ per lane were separated via SDS-PAGE $(10 \%$ gel) and transferred to polyvinylidene difluoride membranes, which were blocked with a blocking solution (5\% bovine serum albumin and 0.1\% Tween 20 in TBS; Beyotime Institute Biotechnology) for $1 \mathrm{~h}$ at room temperature. Subsequently, the membrane was incubated with anti-HIF- $1 \alpha$ primary antibodies (1:2,000; ab187524; Abcam) overnight at $4^{\circ} \mathrm{C}$, followed by incubation with horseradish peroxidase-conjugated secondary antibodies (1:2,000; 31460; Thermo Fisher Scientific, Inc.). The bands were visualized using an enhanced chemiluminescence detection system (ChemiDoc XRS; Bio-Rad Laboratories, Inc., Hercules, CA, USA) and were quantified using Quantity One software (version 4.6; Bio-Rad Laboratories, Inc.).

Detection of hypoxia in tumors. For the detection of hypoxia, mice were divided into two groups, the control mice $(n=20)$ were treated with PBS, and the treatment mice $(n=20)$ were treated with Endostar. Following treatment, each mouse was injected intraperitoneally with $60 \mathrm{mg} / \mathrm{kg}$ pimonidazole (Hypoxyprobe). After $1 \mathrm{~h}$, tumor samples were collected, frozen in liquid nitrogen and cryosectioned into $10-\mu \mathrm{m}$ sections, and immunohistochemical analysis was performed as above.

In vitro cell viability assays. The viability of SW620 cells was measured using a Cell Counting Kit-8 (CCK-8; Dojindo Molecular Technologies, Inc., Kumamoto, Japan). Briefly, cells were seeded in 96 -well plates $\left(10^{4}\right.$ cells per well $)$ and treated with Endostar (0, 2550 and $100 \mathrm{ug} / \mathrm{ml})$ or PBS for $24 \mathrm{~h}$ at $37^{\circ} \mathrm{C}$ in $5 \% \mathrm{CO}_{2}$. CCK-8 reagent $(10 \mu \mathrm{l})$ was added to each well, and the plates were incubated at $37^{\circ} \mathrm{C}$ in $5 \% \mathrm{CO}_{2}$ for another $2 \mathrm{~h}$. Absorbance was measured at $450 \mathrm{~nm}$ using a microplate reader (BioTek Instruments, Inc., Winooski, VT, USA).

Statistical analysis. Data were presented as the mean \pm standard error of the mean. Different groups were compared by one-way analysis of variance with Bonferroni post-hoc test or unpaired Student's t-tests. Statistical analyses were performed using GraphPad Prism 5.0 software (GraphPad Software, Inc., La Jolla, CA, USA). Each experiment was conducted at least three times. $\mathrm{P}<0.05$ was considered to indicate a statistically significant difference.

\section{Results}

Short-term rhES treatment induces a 'time window' of tumor vascular normalization. The 'time window' of tumor vascular normalization has been suggested to occur on days 4 to 6 following anti-angiogenic treatment; in most cases, maximum tumor vascular normalization occurred on day $7(15,21)$. To examine the association between TSP-1 expression and vascular normalization, the vascular appearance, including the density and coverage of pericytes, in a SW620 xenograft tumor model was dynamically monitored. CD31 was used as a biomarker of vascular endothelial cells to detect alterations in vascular morphology and vessel density. The results of immunofluorescence staining demonstrated that vessel density significantly increased by day 12 compared with day 0 in the control group $(\mathrm{P}<0.05)$. In rhES-treated mice, the vessel density was significantly decreased at day $4(\mathrm{P}<0.05)$ and low vessel density persisted until day 6 (Fig. 1A-D).

Pericytes surround the vascular endothelium and are often used as an indicator of blood vessel function and integrity, and can be detected via $\alpha$-SMA expression (22). The present study observed that the pericyte coverage of tumor vessels was increased in the rhES-treated group, particularly at day 6 $(\mathrm{P}<0.05)$, while there were no significant differences in control mice (Fig. 1E-H). Collectively, these data indicated that anti-angiogenic treatment using rhES may facilitate a transient vascular normalization 'time window' that occurs approximately between day 4 and 6 following treatment. The ratio of $\alpha$-SMA-positive to CD31-positive areas has often been used to identify the vessel maturity index $(11,22)$. Analysis of vascular maturity (an important indicator of tumor vessel integrity) in the present study revealed that maturity significantly increased at days 4 and 6 in rhES-treated mice, compared with day 0 $(\mathrm{P}<0.001)$, while control mice exhibited no significant difference. These results suggested that rhES may transiently normalize tumor vessels; however, the maturity appeared to reverse to baseline at day 12 . This may potentially be due to the continuous use of rhES resulting in abnormal vessel development by subsequent over-pruning (Fig. 1I and J).

Tumor tissue exhibits reduced hypoxia during the 'time window' of tumor vascular normalization. Rapid growth of solid tumors is often accompanied by hypoxia, which increases the degree of malignancy and reduces the efficacy of therapy (9). The remission of hypoxia is an additional feature of 
A
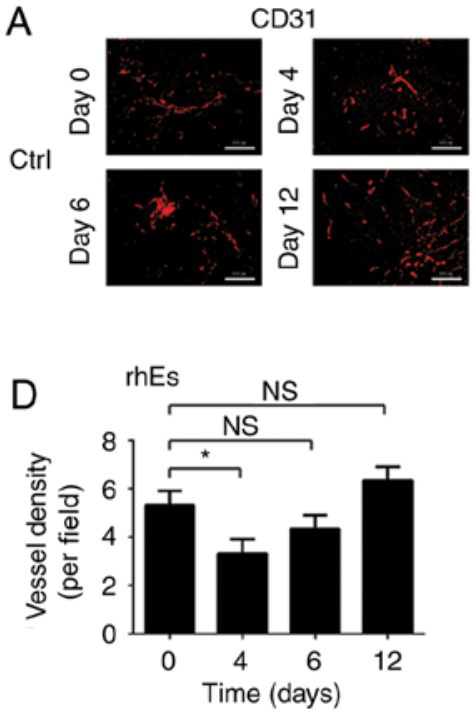

G

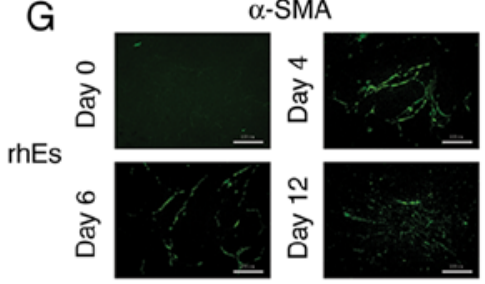

$\mathrm{B}$

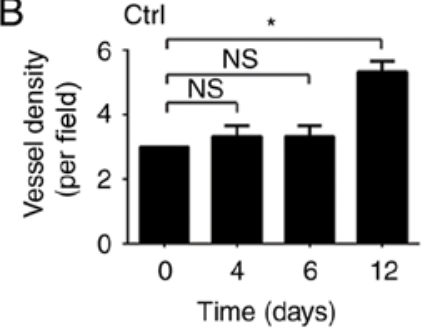

E

Ctrl

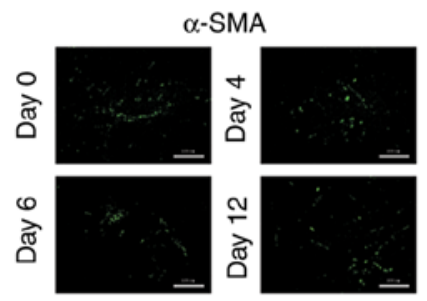

C

rhEs
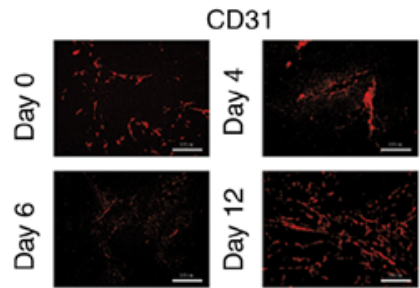

$\mathrm{H}$

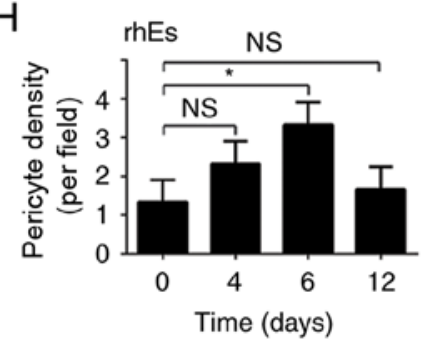

$\mathrm{F}$

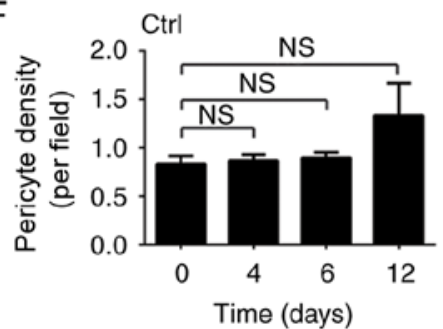

।
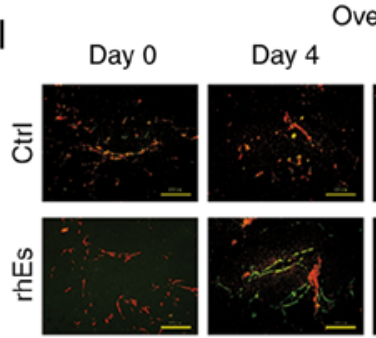

Overlay
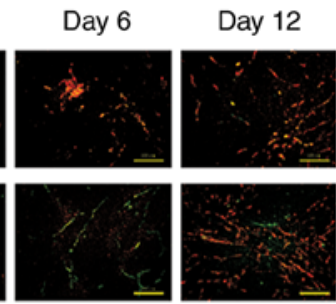

$J$

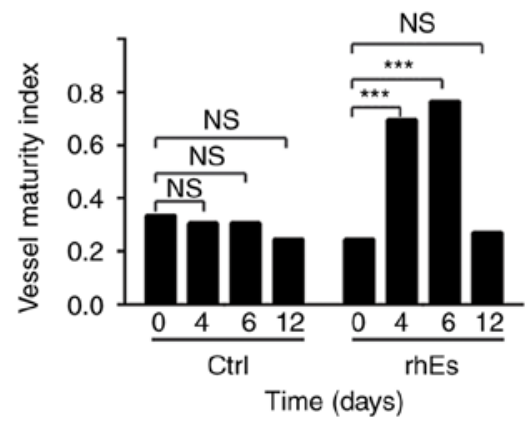

Figure 1. (A-J) rhES-induced tumor vasculature normalization in SW620-bearing mice. (A and C) Immunofluorescence for CD31 (red). Scale bar, $100 \mu \mathrm{m}$ Representative images of SW620 tumor sections from (A) control and (C) rhES-treated groups (after 0, 4, 6 and 12 days, $n=20$. (B and D) Quantification of vessel density. (B) A significant increase in vessel density was observed on day 12 in the control group. (D) A significant decrease at day 4 was observed in the rhES-treated group. (E and G) Immunofluorescence for $\alpha$-SMA (green) in tumors of the (E) control and (G) rhES-treated groups (after 0, 4, 6 and 12 days, $\mathrm{n}=20$. Scale bar, $100 \mu \mathrm{m}$. (F and H) Quantification of pericytes of vessels marked by $\alpha$-SMA. (F) No differences in pericyte coverage was observed in the control group. (H) A significant increase at day 6 was observed in the rhES-treated group. (I) Overlay of (A and E) and (C and G). Vessel maturity index was determined as the ratio of $\alpha$-SMA-positive to CD31-positive areas. $(\mathrm{J})$ There were no differences in the vessel maturity index among days $0-12$ in the control group. A significant increase in the vessel maturity index of rhES-treated tumors was observed at days 4 and 6 , but was restored by day 12 . All quantitative data are presented as the mean \pm standard error of the mean. ${ }^{*} \mathrm{P}<0.05 ;{ }^{* * *} \mathrm{P}<0.001$. CD, cluster of differentiation; rhES, recombinant human endostatin; $\alpha$-SMA, $\alpha$-smooth muscle actin; Ctrl, control; NS, not significant.

tumor vascular normalization $(9,21)$, and therefore reductions in hypoxia may be applied in the prediction of the 'time window' associated with tumor vascular normalization. To evaluate the oxygen supply, the present study analyzed the pimonidazole staining patterns in tumors. The results revealed that the extent of hypoxia was significantly decreased at 6 days after treatment with rhES $(\mathrm{P}<0.05)$; however, it was observed that hypoxia was increased again after 12 days. $(\mathrm{P}<0.01$; Fig. $2 \mathrm{~A}$ and $\mathrm{B})$. This indicated that the tumor vessels were no longer normalized at day 12. To further detect the recovery of oxygen in tumors, HIF-1 $\alpha$ levels in tumor sections were analyzed by western blot analysis. Compared with the expression levels at day 0 , significant downregulation of HIF-1 $\alpha$ was observed on days 4 and $6(\mathrm{P}<0.05)$, while no difference was observed on day 12 (Fig. 2E and F). In addition, the necrotic areas of tumor sections were analyzed, since this is established to be associated with hypoxia (Fig. 2G-J). The necrotic area increased continuously in control mice over the 12 day observation period; meanwhile, in the rhES treatment group, a significant decrease at day 6 , but an increase at day 12 was observed (both $\mathrm{P}<0.05$ ), which appeared associated with the levels of hypoxia induction. Collectively, these results demonstrated that the reduction in hypoxia may be temporary, and the duration of reduced hypoxia may coincide with the suggested 'time window'. 
A

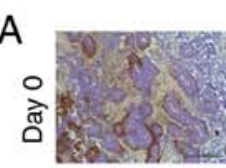

Ctrl
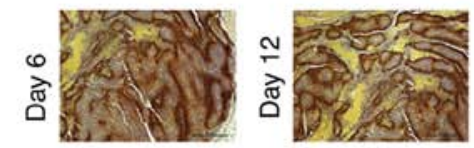

C

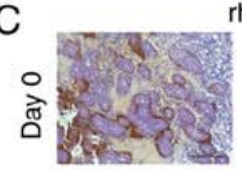

rhEs

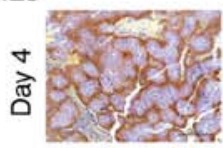

तั
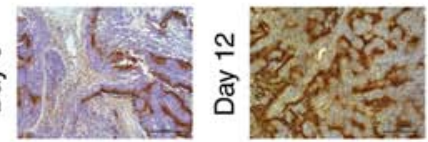

E
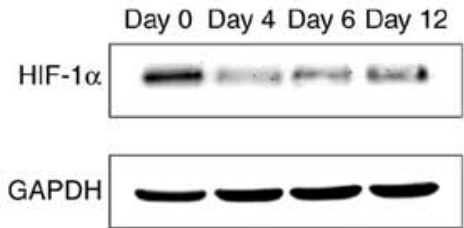

G

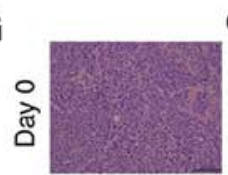

Ctrl
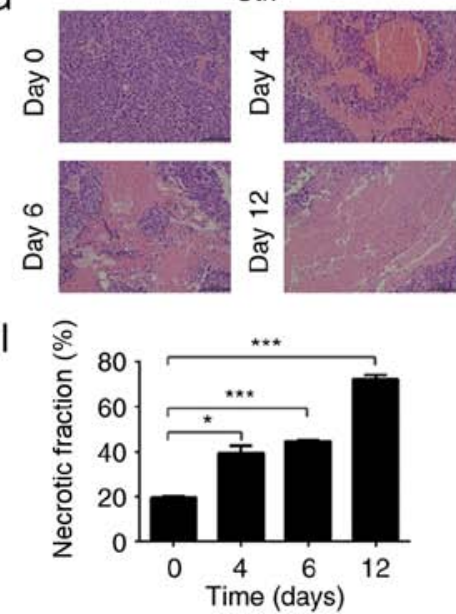

B

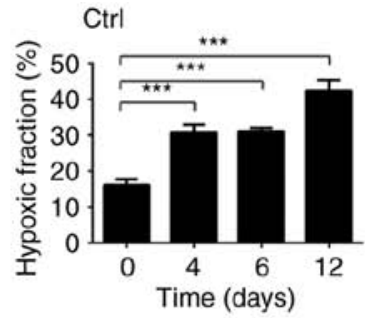

D
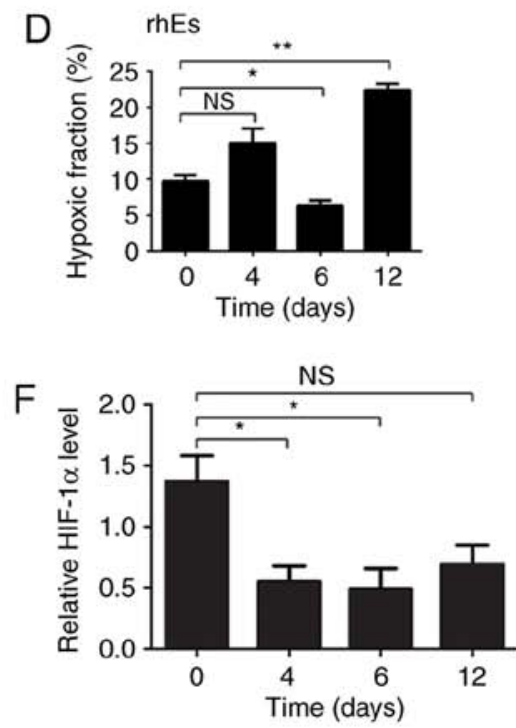

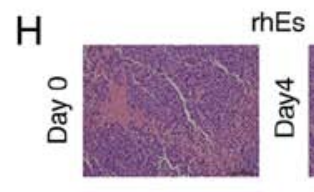
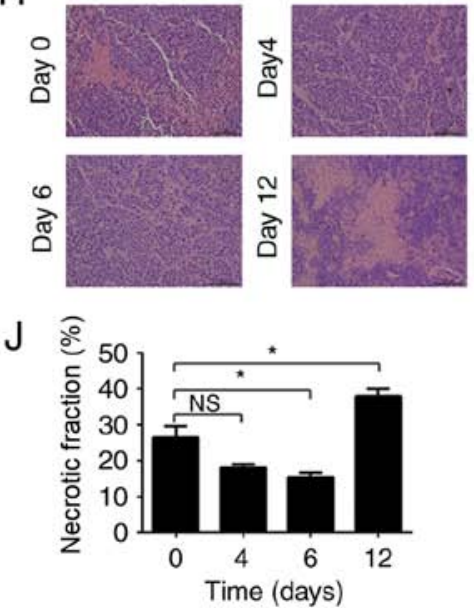

Figure 2. Decrease in tumor hypoxia during tumor vasculature normalization. Tumor tissue from mice bearing primary xenograft tumors were collected, and pimonidazole staining of the tumor tissue was conducted to indicate the hypoxic tissue. (A and C) Hypoxia marker staining by pimonidazole (brown) in sections from (A) control mice (after 0, 4, 6 and 12 days; $n=20$ and (C) rhES-treated mice (after 0, 4, 6 and 12 days; $n=20$ ). Scale bar, 500 $\mu$ m. (B and D) Quantification of pimonidazole-positive areas as a percentage of the whole tumor area. (B) Within the control mice, the hypoxic areas increased significantly at days 4,6 and 12 . (D) Hypoxia was significantly decreased at day 6, but increased at day 12 in the rhES-treated mice. (E) HIF-1 $\alpha$ expression in the tumor sections of rhES-treated mice was significantly decreased at days 4 and 6, indicating reductions in tumor hypoxia; (F) no significant change in HIF-1 $\alpha$ expression was detected at day 12 . $(\mathrm{G}$ and $\mathrm{H})$ Necrotic areas in tumors of the $(\mathrm{G})$ control and $(\mathrm{H})$ rhES-treated mice (after $0,4,6$ and 12 days; hematoxylin and eosin staining). Scale bar, $100 \mu \mathrm{m}$. (I and J) Percentage of necrosis of the whole tumor area. (I) Necrosis was significantly increased at days 4, 6 and 12 in control mice. (J) Necrosis was significantly decreased at day 6 , but significantly increased at day 12 in rhES-treated mice $(\mathrm{J})$. All quantitative data are presented as the mean \pm standard error of the mean. ${ }^{*} \mathrm{P}<0.05 ;{ }^{* *} \mathrm{P}<0.01 ;{ }^{* * *} \mathrm{P}<0.001$. HIF-1 $\alpha$, hypoxia inducible factor- $1 \alpha$; rhES, rhES, recombinant human endostatin; Ctrl, control; NS, not significant.

Consistent with the 'abnormal-normal-abnormal' transformation of tumor vasculature, plasma TSP-1 expression levels fluctuate dynamically. The present study hypothesized that the oxygen content available to tumors is associated with the tumor vascular normalization 'time window', the analysis of hypoxia may theoretically indicate alterations in the duration of the window. However, current methods to detect tumor hypoxia are not readily applicable in the clinic. Therefore, the present study aimed to identify an effective biomarker of the tumor vascular normalization 'time window' based on secreted factors in the blood plasma.

TSP-1 is an endogenous anti-angiogenic factor associated with hypoxia. Therefore, the present study investigated whether tumor vascular normalization may induce corresponding 
A

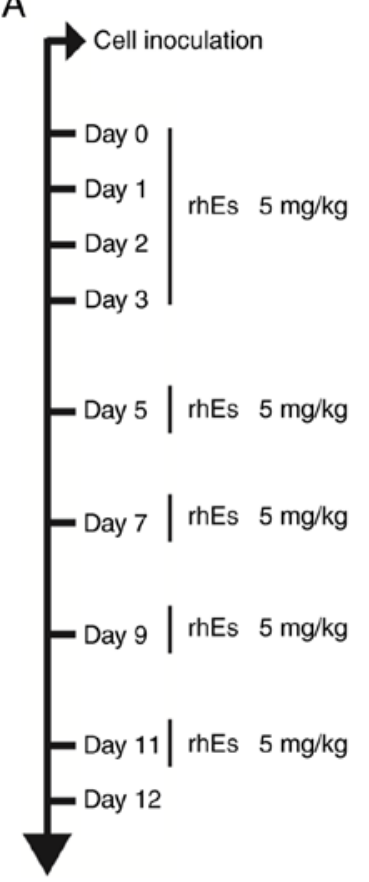

B

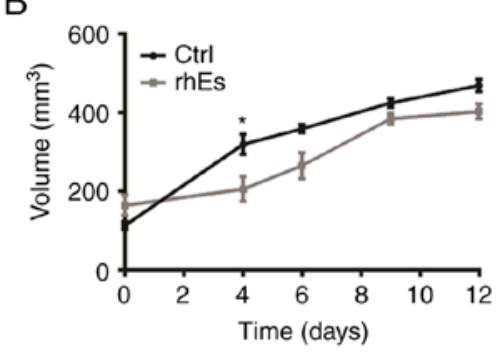

D

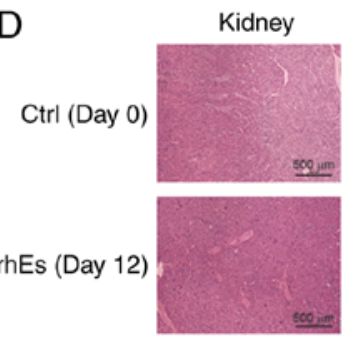

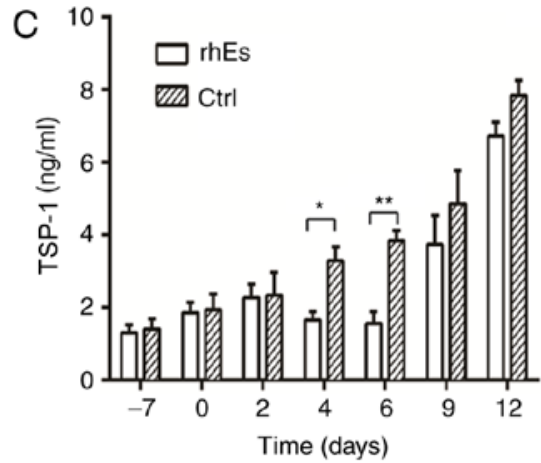

E

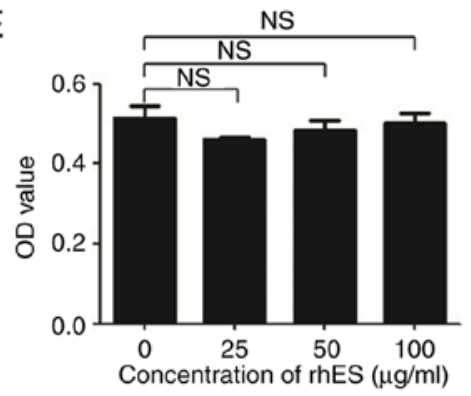

Figure 3. Consistent with the 'abnormal-normal-abnormal' transformation of tumor vasculature, plasma TSP-1 levels fluctuate dynamically. (A) Schedule of rhES therapy. (B) Tumor growth curves. Tumor volumes from mice treated with rhES compared with those of the control. No significant differences in tumor volumes between the treatment and control groups were observed on days 0-12, except on day 4. (C) TSP-1 levels in the plasma of control and rhES-treated mice exhibited a significant difference on days 4 and 6. (D) Organ toxicity analysis of the kidney and liver from control and rhES-treated mice on day 0 and 12 $(\mathrm{n}=20)$. No notable alterations in the structure of the liver or kidney were observed (hematoxylin and eosin staining). All quantitative data are presented as the mean \pm standard error the mean. (E) Cell Counting Kit- 8 results revealed that rhES had no effect on the proliferation of $\mathrm{SW} 620$ cells. ${ }^{*} \mathrm{P}<0.05$; ${ }^{* *} \mathrm{P}<0.01$. rhES, rhES, recombinant human endostatin; TSP-1, thrombospondin-1; Ctrl, control; NS, not significant; OD, optical density.

alterations in the levels of TSP-1 in the plasma. First, rhES was applied to induce tumor vasculature normalization. To eliminate the effect of rhES itself on plasma TSP-1, the drug was used continuously (Fig. 3A), which may generate abnormal tumor vasculature due to over-pruning. The tumor volumes did not differ significantly between the control and rhES-treated mice, except on day 4, which suggested that angiogenesis may have been controlled (Fig. 3B).

Following treatment with rhES, the levels of TSP-1 in the plasma gradually decreased from day 2 to 6 , after which TSP-1 level exhibited a gradual increasing trend (Fig. 3C). This trend was consistent with the amelioration of the morphological characteristics of blood vessels; pericytes of vessels were increased, vessel density was decreased, and the improvements in tumor hypoxia observed in our previous experiments, the most direct effect of hypoxia improvement was that the area of hypoxia necrosis in tumor tissue was decreased $(8,9)$. Conversely, TSP-1 levels in the control samples increased during the observation period. In addition, structural alterations in the morphology of the liver and kidney were not observed during treatment with rhES, indicating that rhES may not affect the expression of TSP-1 in other organs (Fig. 3D). Meanwhile, the in vitro cell viability assays demonstrated that rhES only minimally affected SW620 cell proliferation, which suggested that rhES may not notably affect the secretion of TSP-1 by SW620 cells (Fig. 3E). Therefore, alterations in TSP-1 levels may indicate the duration of tumor vasculature normalization, which may be associated with alleviations in hypoxia.

\section{Discussion}

Solid tumors receive nutrients and oxygen via pervasive abnormal blood vessels, which leads to hypoxia and increased interstitial fluid pressure (IFP) in the tumor stroma $(1,23,24)$. These events increase the degree of malignancy, and the risk of invasion and metastasis (25); the efficacy of radiotherapy and chemotherapy is also reduced. Normalization of the tumor vasculature via anti-angiogenic therapy can reduce hypoxia and decrease the degree of IFP (26), which may enhance the effects of traditional anti-cancer therapies (27-30). Unfortunately, the tumor vascular normalization 'time window' is transient, and patients with various types of tumors may exhibit variations in their respective window (31). Therefore, identifying a readily detected and representative biomarker to analyze the 'time window' may be critical in the application of anti-cancer treatment. The findings of the present study suggested that plasma TSP-1 levels following anti-angiogenic treatment may be used as an indicator of the progression of tumor vasculature normalization.

TSP-1 is a glycoprotein secreted from platelets and was the first identified endogenous protein inhibitor of angiogenesis $(16,23)$. TSP-1 has an in vivo half-life of $\sim 9 \mathrm{~h}$ (24), which is beneficial for an endogenous marker. Hypoxia has been reported to increase the secretion of TSP-1 (25) and promote angiogenesis in tumors $(32,33)$. The present study suggested that as tumor hypoxia decreases, the expression levels of TSP-1 in the plasma may change accordingly. Notably, it was demonstrated that plasma TSP-1 expression levels decreased from day 4 to day 6 following treatment with rhES; these levels returned to 
those of the control group from day 9. Alterations in the levels of TSP-1 were accompanied by changes in vascular morphology, which exhibited features of normalization. In accordance with these changes, HIF-1 $\alpha$ expression in the tumor tissue reflected a decrease in hypoxia. Additionally, the control and rhES-treated groups exhibited gradual increases in the expression of TSP-1, which was accompanied with increases in tumor volume under hypoxia. However, plasma levels of TSP-1 fluctuated in the rhES-treated group but not in the control mice. RhES, a vascular angiogenesis-disrupting agent, has been reported to exhibit anti-tumor effects following treatment for 3 weeks (34). However, in the present study, tumor volumes overall lacked significant differences between the control- and rhES-treated mice. This may be due to the short duration of the $<1$ week of induction of tumor vasculature normalization by rhES.

To further investigate the inhibitory effects of rhES on TSP-1 expression in vivo, continuous administration of treatment was conducted in the present study. This method of treatment may have produced effective decreases in hypoxia during the 'time window' and increased hypoxia in areas where tumor vessels were excessively depleted. Under these circumstances, dynamic alterations in TSP-1 expression levels were observed. Furthermore, it was observed that rhES did not notably affect the proliferation of SW620 cells and did not alter the structure of other organs. This suggested that the reduction in TSP-1 expression levels may be due to a negative feedback mechanism following increased oxygen content, which improved during vascular normalization, rather than inhibiting cell proliferation or via other underlying mechanisms.

Tissue puncture examinations can measure changes in IFP for analysis of the 'time window'; however, tissue puncture can cause damage to the body, restricting the use of this method. Furthermore, advanced 'vasculature' MRI techniques may be used to evaluate the structural and functional parameters of blood vessels over time, a possibility that has been researched previously (35). Once identified, the anti-angiogenic 'time window', the time from the initiation of tumor vasculature normalization to the end, may be applied in the design of concomitant chemotherapy or radiotherapy regimens to enhance therapeutic effects, avoid unnecessary drug toxicities, and possibly reduce costs. Furthermore, this information may provide an opportunity to identify patients who may be sensitive to anti-angiogenic drugs, but not to mass medication. In the present study, tumor vasculature normalization was investigated via the analysis of TSP-1 levels, a readily detected factor that circulates in the blood.

In conclusion, TSP-1 expression increased in response to hypoxia, but decreased when hypoxia was alleviated in the 'time window' of tumor vasculature normalization, induced by rhES treatment in SW620-xenograft mice. However, whether the 'time window' induced by other anti-angiogenic drugs, including bevacizumab and cediranib, may be associated with TSP-1 levels requires further investigation. In addition, the efficacy of TSP-1 expression as a biomarker of the 'time window' for a variety of anti-angiogenic drugs in the clinic should be validated in the future.

\section{Acknowledgements}

Not applicable.

\section{Funding}

The present study was supported by the National Natural Science Foundation of China (grant no. 81472849), the Guangdong Natural Science Research (grant no. 2014A030313383) and the Guangdong High-level University Construction Fund for Jinan University (grant no. 88016013034).

\section{Availability of data and materials}

The datasets used and analyzed during the present study are available from the corresponding author upon reasonable request.

\section{Authors' contributions}

WY and YP conceived the study and wrote the manuscript. WY, FP and WL collected and analyzed the primary data. XY, $\mathrm{XZ}$ and LQ provided technical support with the experiments, revised the manuscript and gave final approval of the version to be published. All authors read and approved the final manuscript.

\section{Ethics approval and consent to participate}

The research was approved by the Laboratory Animal Ethics Committee of Jinan University (Guangzhou, China).

\section{Patient consent for publication}

Not applicable.

\section{Competing interests}

The authors declare that they have no competing interests.

\section{References}

1. Folkman J: Tumor angiogenesis: Therapeutic implications. N Engl J Med 285: 1182-1186, 1971.

2. Rupaimoole R, Ivan C, Yang D, Gharpure KM, Wu SY, Pecot CV, Previs RA, Nagaraja AS, Armaiz-Pena GN, McGuire M, et al: Hypoxia-upregulated microRNA-630 targets Dicer, leading to increased tumor progression. Oncogene 35: 4312-4320, 2016.

3. Adapala RK, Thoppil RJ, Ghosh K, Cappelli HC, Dudley AC, Paruchuri S, Keshamouni V, Klagsbrun M, Meszaros JG Chilian WM, et al: Activation of mechanosensitive ion channel TRPV4 normalizes tumor vasculature and improves cancer therapy. Oncogene 35: 314-322, 2016.

4. Shin DH, Choi YJ and Park JW: SIRT1 and AMPK mediate hypoxia-induced resistance of non-small cell lung cancers to cisplatin and doxorubicin. Cancer Res 74: 298-308, 2014.

5. Sen A, Capitano ML, Spernyak JA, Schueckler JT, Thomas S, Singh AK, Evans SS, Hylander BL and Repasky EA: Mild elevation of body temperature reduces tumor interstitial fluid pressure and hypoxia and enhances efficacy of radiotherapy in murine tumor models. Cancer Res 71: 3872-3880, 2011.

6. Ashton TM, Fokas E, Kunz-Schughart LA, Folkes LK, Anbalagan S, Huether M Kelly CJ, Pirovano G, Buffa FM, Hammond EM, et al: The anti-malarial atovaquone increases radiosensitivity by alleviating tumour hypoxia. Nat Commun 7: 12308, 2016.

7. Meijer TW, Kaanders JH, Span PN and Bussink J: Targeting hypoxia, HIF-1, and tumor glucose metabolism to improve radiotherapy efficacy. Clin Cancer Res 18: 5585-5594, 2012.

8. Zhang HM, Ren Y, Tang XJ, Wang K, Liu Y, Zhang L, Li X, Liu P, Zhao C and He J: Vascular normalization induced by sinomenine hydrochloride results in suppressed mammary tumor growth and metastasis. Sci Rep 5: 8888, 2015. 
9. Jain RK: Normalizing tumor vasculature with anti-angiogenic therapy: A new paradigm for combination therapy. Nat Med 7: 987-989, 2001.

10. Chatterjee S, Wieczorek C, Schöttle J, Siobal M, Hinze Y, Franz T, Florin A, Adamczak J, Heukamp LC, Neumaier B and Ullrich RT: Transient antiangiogenic treatment improves delivery of cytotoxic compounds and therapeutic outcome in lung cancer. Cancer Res 74: 2816-2824, 2014.

11. Ader I, Gstalder C, Bouquerel P, Golzio M, Andrieu G, Zalvidea S, Richard S, Sabbadini RA, Malavaud B and Cuvillier O. Neutralizing S1P inhibits intratumoral hypoxia, induces vascular remodelling and sensitizes to chemotherapy in prostate cancer. Oncotarget 6: 13803-13821, 2015.

12. Goel S, Duda DG, Xu L, Munn LL, Boucher Y, Fukumura D and Jain RK: Normalization of the vasculature for treatment of cancer and other diseases. Physiol Rev 91: 1071-1121, 2011.

13. Schneider BP, Wang M, Radovich M, Sledge GW, Badve S, Thor A, Flockhart DA, Hancock B, Davidson N, Gralow J, et al: Association of vascular endothelial growth factor and vascular endothelial growth factor receptor- 2 genetic polymorphisms with outcome in a trial of paclitaxel compared with paclitaxel plus bevacizumab in advanced breast cancer: ECOG 2100. J Clin Oncol 26: 4672-4678, 2008.

14. Schneider Duda DG, Willett CG, Ancukiewicz M, di Tomaso E, Shah M, Czito BG, Bentley R, Poleski M, Lauwers GY, Carroll M, et al: Plasma soluble VEGFR-1 is a potential dual biomarker of response and toxicity for bevacizumab with chemoradiation in locally advanced rectal cancer. Oncologist 15 : 577-583, 2010

15. Lassau N, Coiffier B, Kind M, Vilgrain V, Lacroix J, Cuinet M, Taieb S, Aziza R, Sarran A, Labbe-Devilliers C, et al: Selection of an early biomarker for vascular normalization using dynamic contrast-enhanced ultrasonography to predict outcomes of metastatic patients treated with bevacizumab. Ann Oncol 27: 1922-1928, 2016.

16. Good DJ, Polverini PJ, Rastinejad F, Le Beau MM, Lemons RS, Frazier WA and Bouck NP: A tumor suppressor-dependent inhibitor of angiogenesis is immunologically and functionally indistinguishable from a fragment of thrombospondin. Proc Natl Acad Sci USA 87: 6624-6628, 1990.

17. Takahashi K, Sumarriva K, Kim R, Jiang R, Brantley-Sieders DM, Chen J, Mernaugh RL and Takahashi T: Determination of the CD148-interacting region in thrombospondin-1. PLoS One 11: e0154916, 2016.

18. Hamano Y, Sugimoto H, Soubasakos MA, Kieran M, Olsen BR, Lawler J, Sudhakar A and Kalluri R: Thrombospondin-1 associated with tumor microenvironment contributes to low-dose cyclophosphamide-mediated endothelial cell apoptosis and tumor growth suppression. Cancer Res 64: 1570-1574, 2004.

19. Bocci G, Francia G, Man S, Lawler J and Kerbel RS: Thrombospondin 1, a mediator of the antiangiogenic effects of low-dose metronomic chemotherapy. Proc Natl Acad Sci USA 100: 12917-12922, 2003.

20. Firlej V, Mathieu JR, Gilbert C, Lemonnier L, Nakhlé J, Gallou-Kabani C, Guarmit B, Morin A, Prevarskaya N, Delongchamps NB and Cabon F: Thrombospondin-1 triggers cell migration and development of advanced prostate tumors. Cancer Res 71: 7649-7658, 2011.

21. Ohta M, Kawabata T, Yamamoto M, Tanaka T, Kikuchi H, Hiramatsu Y, Kamiya K, Baba M and Konno H: TSU68, an antiangiogenic receptor tyrosine kinase inhibitor, induces tumor vascular normalization in a human cancer xenograft nude mouse model. Surg Today 39: 1046-1053, 2009.

22. Meng MB, Zaorsky NG, Deng L, Wang HH, Chao J, Zhao LJ, Yuan ZY and Ping W: Pericytes: Adouble-edged sword in cancer therapy. Future Oncol 11: 169-179, 2015.
23. Gaustad JV, Simonsen TG, Andersen LM and Rofstad EK: Thrombospondin-1 domain-containing peptide properdistatin improves vascular function in human melanoma xenografts. Microvasc Res 98: 159-165, 2015.

24. Dawes J, Clemetson KJ, Gogstad GO, McGregor J, Clezardin P, Prowse CV and Pepper DS: A radioimmunoassay for thrombospondin, used in a comparative study of thrombospondin, beta-thromboglobulin and platelet factor 4 in healthy volunteers. Thromb Res 29: 569-581, 1983.

25. Phelan MW, Forman LW, Perrine SP and Faller DV: Hypoxia increases thrombospondin-1 transcript and protein in cultured endothelial cells. J Lab Clin Med 132: 519-529, 1998.

26. Jain RK: Normalization of tumor vasculature: An emerging concept in antiangiogenic therapy. Science 307: 58-62, 2005.

27. Giantonio BJ, Catalano PJ, Meropol NJ, O'Dwyer PJ, Mitchell EP, Alberts SR, Schwartz MA and Benson AB III; Eastern Cooperative Oncology Group Study E3200: Bevacizumab in combination with oxaliplatin, fluorouracil, and leucovorin (FOLFOX4) for previously treated metastatic colorectal cancer: Results from the Eastern cooperative oncology group study E3200. J Clin Oncol 25: 1539-1544, 2007.

28. Browder T, Butterfield CE, Kräling BM, Shi B, Marshall B, O'Reilly MS and Folkman J: Antiangiogenic scheduling of chemotherapy improves efficacy against experimental drug-resistant cancer. Cancer Res 60: 1878-1886, 2000.

29. Segers J, Di Fazio V, Ansiaux R, Martinive P, Feron O, Wallemacq P and Gallez B: Potentiation of cyclophosphamide chemotherapy using the anti-angiogenic drug thalidomide: Importance of optimal scheduling to exploit the "normalization' window of the tumor vasculature. Cancer Lett 244: 129-135, 2006.

30. Dickson PV, Hamner JB, Sims TL, Fraga CH, Ng CY Rajasekeran S, Hagedorn NL, McCarville MB, Stewart CF and Davidoff AM: Bevacizumab-induced transient remodeling of the vasculature in neuroblastoma xenografts results in improved delivery and efficacy of systemically administered chemotherapy. Clin Cancer Res 13: 3942-3950, 2007.

31. Jain RK, Duda DG, Willett CG, Sahani DV, Zhu AX, Loeffler JS, Batchelor TT and Sorensen AG: Biomarkers of response and resistance to antiangiogenic therapy. Nat Rev Clin Oncol 6: 327-338, 2009.

32. Facciabene A, Peng XH, Hagemann IS, Balint K, Barchetti A, Wang LP, Gimotty PA, Gilks CB, Lal P, Zhang L and Coukos G: Tumour hypoxia promotes tolerance and angiogenesis via CCL28 and T-(reg) cells. Nature 475: 226-230, 2011.

33. Chouaib S, Messai Y, Couve S, Escudier B, Hasmim M and Noman MZ: Hypoxia promotes tumor growth in linking angiogenesis to immune escape. Front Immunol 3: 21, 2012.

34. Jia Y, Liu M, Huang W, Wang Z, He Y, Wu J, Ren S, Ju Y, Geng $\mathrm{R}$ and Li $\mathrm{Z}$ : Recombinant human endostatin endostar inhibits tumor growth and metastasis in a mouse xenograft model of colon cancer. Pathol Oncol Res 18: 315-323, 2012.

35. Sorensen AG,BatchelorTT,Zhang WT, Chen PJ, Yeo P,Wang MY Jennings D, Wen PY, Lahdenranta J, Ancukiewicz M, et al: A 'Vascular Normalization Index' as potential mechanistic biomarker to predict survival after a single dose of cediranib in recurrent glioblastoma patients. Cancer Res 69: 5296-5300, 2009.

This work is licensed under a Creative Commons Attribution-NonCommercial-NoDerivatives 4.0 International (CC BY-NC-ND 4.0) License. 